Golden Gate University School of Law GGU Law Digital Commons

1984

\title{
L'HUMANITÉ EN TANT QU'ÉLÉMENT CONTRIBUANT AU DÉVELOPPEMENT PROGRESSIF DU DROIT INTERNATIONAL CONTEMPORAIN
}

Sompong Sucharitkul

Golden Gate University School of Law, ssucharitkul@ggu.edu

Follow this and additional works at: http://digitalcommons.law.ggu.edu/pubs

Part of the International Law Commons

\section{Recommended Citation}

Sucharitkul, Sompong, "L'HUMANITÉ EN TANT QU'ÉLÉMENT CONTRIBUANT AU DÉVELOPPEMENT PROGRESSIF DU DROIT INTERNATIONAL CONTEMPORAIN" (1984). Publications. Paper 546.

http://digitalcommons.law.ggu.edu/pubs/546 


\section{L'AVENIR \\ DU DROIT INTERNATIONAL DANS UN MONDE MULTICULTUREL}

COLLOQUE 1983

3. L'HUMANITÉ EN TANT QU'ÉLÉMENT CONTRIBUANT AU DÉVELOPPEMENT PROGRESSIF DU DROIT INTERNATIONAL CONTEMPORAIN

Sompong SUCHARITKUL

(Tiré à part)

1984 


\section{Distributors:}

for the United States and Canada

Kluwer Boston. Inc.

190 Old Derby Street

Hingham. MA 02043

USA

for all other countries

Kluwer Academic Publishers Group Distribution Center

P.O. Box 322

$3300 \mathrm{AH}$ Dordrecht

The Netherlands
ACADEMIE DE DROIT INTERNATIONAL DE LA HAYE

HAGUE ACADEMY OF INTERNATIONAL LAW.
UNIVERSITE

DES NATIONS UNIES

UNITED NATIONS

UNIVERSITY

\title{
L'AVENIR DU DROIT INTERNATIONAL DANS UN MONDE MULTICULTUREL
}

\author{
THE FUTURE OF \\ INTERNATIONAL LAW IN A \\ MULTICULTURAL WORLD
}

COLlOQUE,

LA HAYE, 17-19 NOVEMBRE 1983

WORKSHOP

THE HAGUE, 17-19 NOVEMBER 1983

Préparé par/Edited by : René-Jean DUPUY 


\section{LISTE DES PARTICIPANTS \\ LIST OF PARTICIPANTS}

Anand, R. P.,

Barberis, J.,

Bardonnet, D.,

Bedjaoui, $M$., Ben Achour, $Y$.,

Ben Allal, $M$.,

Benda-Beckmann, E. A. Von

Bozeman, A. B.,

Broches, $A$.,

Cassese, $A$.,

Ch'en, $P$.,

Duanmu, $Z$.,

David, $R$,

Derrett, J. D. M.,

Diop, A.,

Dupuy, R.-J.,

Elias, T. O.,

Ewald, $F$.,

Flory, M.,

Haquani, $Z$., Johnson The
Professor of International Law at the Jawaharlal Nehru University Studies of New Delhi.

professeur à l'Université catholique de Buenos Aires.

professeur à l'Université de droit, d'économie et de sciences sociales de Paris.

juge à la Cour internationale de Justice

professeur à la faculté de droit de l'Université de Tunis.

directeur de l'Ecole nationale d'administration publique, Rabat.

professeur à l'U
Leeuwenborch $》$. Lawrence College, Bronxville, NY.

Counsellor at Law, Former Vice President and General Counsel of the World Bank.

Professor of International Law at the University of Florence.

Professor, Department of Law, School of Oriental and African Studies, London.

doyen de la faculté de droit de l'Université Sun Yat-Sen Guangzhou de Canton.

professeur honoraire aux facultés de droit de Paris et d'Aix-en-Provence.

Professor of Oriental Law at the University of London.

professeur à l'Institut fondamental d'Afrique Noire, Dakar.

professeur au Collège de France, secrétaire général de l'Académie de droit international de La Haye.

président de la Cour internationale de Justice. chercheur au Centre national de la recherche scientifique, Paris.

professeur à la faculté de droit et de science politique d'Aix-Marseille.

Maître assistant associé à l'Université de Paris XI. Khadduri, $M$.,
Ambassador, Legal Advisor of the Sw
nistry of Foreign Affairs, Stockholm.

Professor Emeritus, The Johns Hopkins University, Washington 
Ladreit de Lacharrière, $G$., juge à la Cour internationale de Justice.

Lachs, $M$.

Makarczyk, $J$,

Mbaye, $K$.,

McWhinney, E., Q.C.

Merle, $M$.,

Morozov, P. D.,

Mushakoji, $K$.,

Nagendra Singh Nahlik, S. E.

Oda, $S$,

Philip, A.,

Ploman, E. W.

Preiser, $W$.,

Rouveroy van Nieuwaal E. A.B. van,

Rysiak, G.,

Saba, $H$.,

Sanson, H., S.J.

Sarin, $M . L$.

Sette-Camara, J.,

Solomon, E.

Sucharitkul, S.

Trapero-Ballestero, $A$.,

Truyol Serra, A.,

Uribe Vargas, $D$. Virally, M.,

Dupuy, R.-J.

\section{Coordinateur/Co-ordinator}

Assistance technique/Technical Assistance

Haquani, $Z$. professeur au Collège de France; secrétaire général de l'Académie de droit international de La Haye.

juge à la Cour internationale de Justice.

Deputy Director, Institute of State and Law,

Professor of International Law and Relations, Simon Fraser University, Burnaby.

professeur au département de science politique Universite de Paris 1.

uge à la Cour internationale de Justice. of the United Nations University.

uge à la Cour internationale de Justice.

uge à la Cour internationale de Justice.

du Curatorium de l'Acadé-

de droit a 'Université de Francfort.

Professor at the University of Leyden, African Prudies Centre.

professeur titulaire à l'Université Jagellonne, ancien sous-directeur général adjoint de l'Unesco. chercheur en sciences sociales au Centre national de la recherche scientifique, Paris.

Jublitute of Paw, University of Giessen.

uge à la Cour internationale de Justice.

directeur d'analyses sociales et économiques,

mbassadeur de Thaïlande à Rome, membre de la Commission du droit international.

Cirecteur de l'enseignement supérieur, Unesco.

membre du Curatorium de l'Académie de droit international de La Haye, professeur à l'Univer-

ambassadeur de Colombie à Paris.

professeur à l'Université de droit, d'économie te sciences sociales de Paris.

maître assistant associé à l'Université de Paris XI.

\section{TABLE DES MATIERES - CONTENTS}

Présentation, par R.-J. Dupuy . . . . . . . . . . . XV

Presentation by R.J. Dupuy . . . . . . . . . . . . XVII

Ouverture du colloque par/Opening of the Workshop by

M. Lachs . . . . . . . . . . . . . . 3

E. W. Ploman ......................

E. Solomon $\ldots \ldots \ldots \ldots \ldots \ldots \ldots \ldots \ldots \ldots \ldots \ldots$

Introduction du sujet, par R.-J. Dupuy . . . . . . . . . . . . 17

Introduction of the subject by R.-J. Dupuy . . . . . . . . . . 25

\section{PREMIÈRE PARTIE \\ APPROCHE GLOBALE}

FIRST PART

GLOBAL APPROACH

\section{Communications}

\section{A. La problématique}

The problems

1. LE POINT DE VUE DU POLITOLOGUE, par M. MERLE ...... 39

2. LE POINT DE VUE DU PHILOSOPHE, par F. EWALD . . . . . . . . 49

I. Du contrat au consensus . . . . . . . . . . . . 50

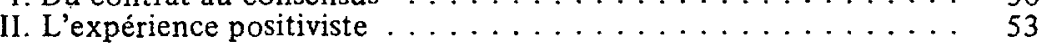

3. LE POINT DE VUE DU SOCIOLOGUE: MODĖLES DE COEXISTENCE DANS LA DIFFERENCE DES CULTURES, par

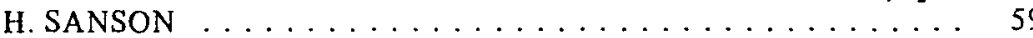

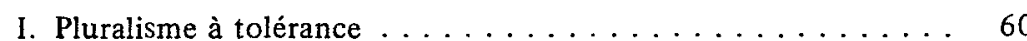

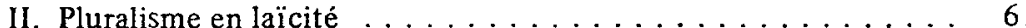

III. Pluralisme d'égalité .......

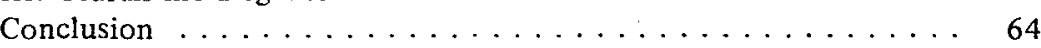

4. LE POINT DE VUE DU JURISTE: LA PRODUCTION ET L'APPLICATION DU DROIT INTERNATIONAL DANS UN MONDE MULTICULTUREL, par G. de LACHARRIÈRE . . . . . . . . .

B. Le facteur multiculturel et le droit international The multicultural factor and international law

1. AN INTRODUCTION TO VARIOUS CULTURAL TRADITIONS OF INTERNATIONAL LAW - A PRELIMINARY ASSESSMENT by A, B. BOZEMAN $\ldots \ldots \ldots$ 
2. THE ROLE OF ASIAN STATES IN THE DEVELOPMENT OF INTERNATIONAL LAW by R. P. ANAND . . . . . . . . . 105

Heterogeneous world society $\ldots \ldots \ldots \ldots \ldots$ Culture and cultural influence . . . . . . . . . . . . . 105 International law, product of European civilization . . . . . . . . 106 Eurocentrism .......................... 107 Crisis in the law of nations $\ldots \ldots \ldots \ldots . \ldots \ldots$ History and cultural traditions of Asian States ignored . . . . . . . 109 International law developed for the protection of European interests 110 Recent challenge to traditional law $\ldots \ldots \ldots \ldots \ldots \ldots \ldots \ldots \ldots$

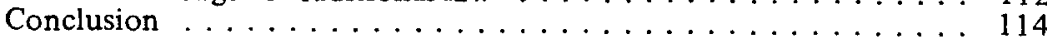

3. THE ASIAN-AFRICAN STATES AND THE DEVELOPMENT OF INTERNATIONAL LAW by M. L. SARIN . . . . . . . . . . 117

I. Introduction .................... 117

II. Divergent opinions on the development of the family of nations and international law $\ldots \ldots \ldots \ldots \ldots \ldots \ldots \ldots \ldots \ldots \ldots \ldots$

III. Europe, Asia and Africa and the development of international law ......................... 120

1. The development from the 19 th century onwards . . . . . . . 123

2. Specific contribution of the Asian-African States: the role of the Asian-African Legal Consultative Committee .......

IV. The impact of cultural factors on the attitudes of States towards

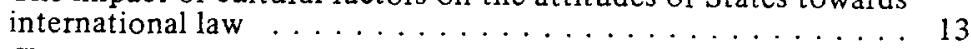
Clash of interest between the industrialized and the developing
countries $\ldots \ldots \ldots \ldots \ldots \ldots \ldots \ldots \ldots \ldots \ldots \ldots \ldots \ldots$

V. Concluding observations .............. 137

4. QUELQUES RÉFLEXIONS SUR LE DROIT ET SUR L'ÉTUDE SOCIO-JURIDIQUE EN AFRIQUE, par E. A. B. VAN ROUVEROY

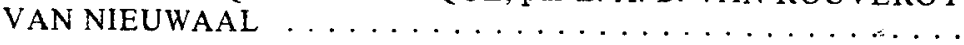

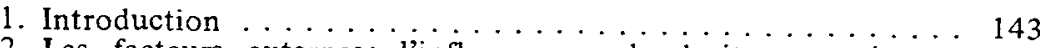
2. Les facteurs externes: linfluence sur le droit coutumier en Afrique

3. Le pluralisme juridique et la réception du droit européen $\ldots \ldots \ldots 145$

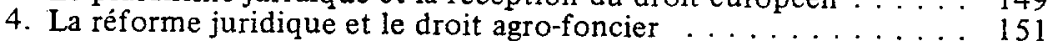

$$
\text { II }
$$

1. Etude sur le droit coutumier africain $\ldots \ldots \ldots \ldots \ldots \ldots$

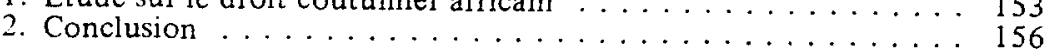

5. ISLAMIC LAW AND INTERNATIONAL LAW bY M. KHADDURI . 157

6. DÉCLARATION ISLAMIQUE UNIVERSELLE DES DROITS DE L'HOMME, par Z. HAQUANI . . . . . . . . . . . . . 163

1. La reconnaissance des droits civils et politiques . . . . . . . . 164

II. La reconnaissance des droits économiques et socio-culturels $\ldots . .164$ III. La reconnaissance des droits de solidarité . . . . . . . . 169 Annexe ......................... 173

II. Résumé des débats . . . . . . . . . . . . . . . 185 Summing up ...................... 201
DEUXIĖME PARTIE

\section{APPROCHE COMPARATIVE}

ET THEMATIQUE

SECOND PART

COMPARATIVE AND

THEMATIC APPROACH

\section{Communications}

A. L'approche comparative

The comparative approach

1. COMPARATIVE INTERNATIONAL LAW: REGIONAL OR SECTORIAL, INTER-SYSTEMIC APPROACHES TO CONTEMPORARY INTERNATIONAL LAW by E. McWHINNEY . . . . . 221

2. THE BASIC CONCEPT OF UNIVERSALITY AND THE DEVELOPMENT OF INTERNATIONAL LAW by NAGENDRA SINGH . 239

1. The raison d'être of international law in the context of a multicultural community of States $\ldots \ldots \ldots \ldots$ 2. The evolutionary growth of the universality of inter-State law and

(i) The principle of exchange of prisoners of war $\ldots \ldots . \cdots 21$

(ii) Rule relating to exchange of bodies of war victims $\ldots \ldots \ldots 243$

(iii) The advent of Christendom in South East Asia and the concept of efficacity of treaty law $\ldots \ldots \ldots \ldots \ldots$
The territorial concept of culture and its jurisdiction over

(iv) The territorial concept of culture and its jurisdiction over individuals and the development of international law ......

(vi) The institution of envoys and emissaries .......... 248

3. The heritage of the past and the future of inter-State law . . . . . 249

4. An analytical assessment of the obstacles, whether cultural or otherwise, in the way of future development of international law 250

5. The future of international law lies in promoting and strengthening the agencies and instrumentalities which stand for its development

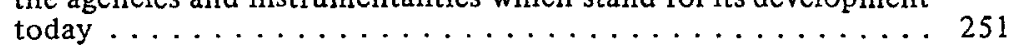

(i) The two aspects concerning the future and the priority of one . 251

(ii) The inter-governmental instrumentalities which stand for the development of international law today .......... 253 (a) The United Nations . . . . . . . . . . . . . 253 (b) Specialized agencies of the United Nations . . . . . . 254

(iii) Non-governmental institutions which stand for promotion of

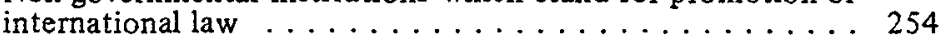

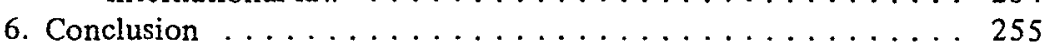

The efficacy of the inter-State law . . . . . . . . . 257

3. DIFFERENT TRENDS OF THE INTERNATIONAL LEGAL SYSTEM OF TODAY bY B. JOHNSON THEUTENBERG $\ldots \ldots \ldots 261$

B. Les concepts

The concepts

1. LE DROIT INTERNATIONAL EST-ILEUROPÉEN? parM.FLORY 287

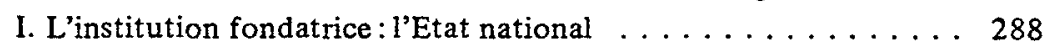




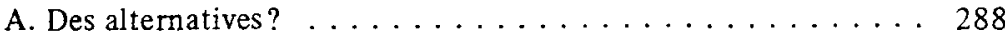

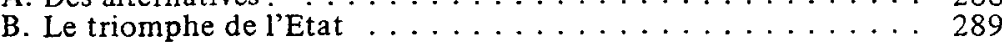

II. Le produit juridique . . . . . . . . . . . . . . . . . . 292

A. L'élaboration formelle du droit international . . . . . . . . . 292

B. Le contenu matériel . . . . . . . . . . . . . . . . . 293

2. FRONTIÈRES ET CULTURES, par D. BARDONNET . . . . . . 299

3. THE PRIMITIVE CHRISTIAN TEACHING CONCERNING VIOLENCE by J. D. M. DERRET . . . . . . . . . . . . . . 311

4. THE CONCEPT OF LAW UPHELD BY WESTERN, SOCIALIST AND DEVELOPING COUNTRIES by A. CASSESE . . . . . . 317

5. LA CHARTE INTERNATIONALE DES DROITS DE L'HOMME, SON ELABORATION ET SON APPLICATION DANS UN MONDE

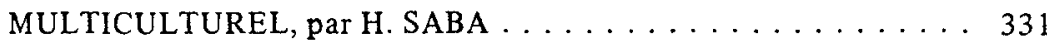

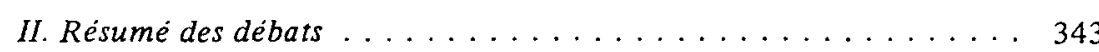

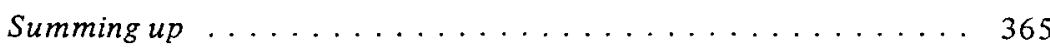

TROISIÈME PARTIE

SOLUTIONS D'AVENIR

THIRD PART

FUTURE SOLUTIONS

\section{Communications}

1. LE DROIT À L'ÉDUCATION ET LE DROIT INTERNATIONAL, pat A. TRAPERO-BALLESTERO . . . . . . . . . . . 391

2. L'ARBITRAGE, SOLUTION D'AVENIR POUR LE DROIT INTERNATIONAL, paI R. DAVID . . . . . . . . . . . . . 401

3. L'HUMANITÉ EN TANT QU'ÉLÉMENT CONTRIBUANT AU DÉVELOPPEMENT PROGRESSIF DU DROIT INTERNATIONAL CONTEMPORAIN, par S. SUCHARITKUL . . . . . . . . . 415

Les pirates ex jure gentium: «hostes generis humani » . . . . . . . 4 420 Un code des crimes contre la paix et la sécurité de l'humanité .... 421 Le caractère humanitaire du nouveau droit international . . . . . . . 423 Le patrimoine commun de l'humanité ............. 425

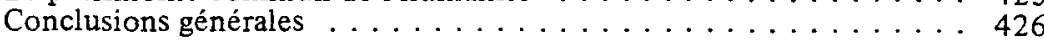

II. Résumé des débats . . . . . . . . . . . . . . . . . . . 433

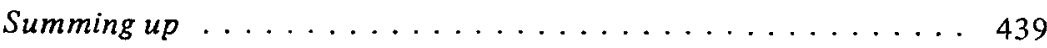

CONCLUSIONS DU COLLOQUE, par R.-J. DUPUY . . . . . . . . . . 447

I. Le constat : multiculturalisme et droit international . . . . . . . . . 449

A. Le droit international comme produit interculturel . . . . . . 450

B. Le droit international face aux conduites culturelles . . . . . . 451

C. Le droit international et les systèmes de valeurs . . . . . . . . 454

II. La prospective : vers un droit de l'humanité? . . . . . . . . . . . . 456 A. Les produits juridiques du concept d'humanité . . . . . . . . 459

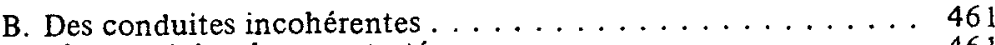
C. L'humanité, valeur contestée ............. 461 CONCLUSIONS OF THE WORKSHOP by R.-J. DUPUY . . . . . . . 469

I. The report : multiculturalism and international law . . . . . . 471

A. International law as an intercultural product . . . . . . 472

A. International law as an intercultural product $\ldots \ldots \ldots \ldots \ldots 4.473$

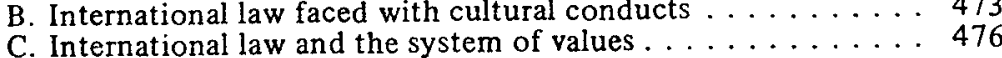

II. The prospect : towards a law of mankind? . . . . . . . . . 478

A. The juridical products of the concept of mankind . . . . . . 481

B. Incoherent conducts . . . . . . . . . . . . . 482

C. Mankind, a challenged value $\ldots \ldots \ldots \ldots \ldots \ldots \ldots \ldots \ldots \ldots$

CLŌTURE DU COLLOQUE, par M. LACHS . . . . . . . . . . . . . 49

CLOSING OF THE WORKSHOP bY M. LACHS . . . . . . . . . . 491 .

\author{
.
}

.

.




\title{
3. L'HUMANITÉ EN TANT QU'ÉLÉMENT CONTRIBUANT AU DÉVELOPPEMENT PROGRESSIF DU DROIT INTERNATIONAL CONTEMPORAIN
}

\author{
Sompong SUCHARITKUL
}

Une question préliminaire qui se pose en abordant l'avenir du droit international dans un monde multiculturel est celle de savoir si le droit international est vraiment disposé à accorder ou à reconnaître un statut juridique quelconque à l'humanité en tant que telle. Or, jusqu'ici l'humanité dans sa totalité, en dépit des distinctions culturelles et malgré les différences ethniques, linguistiques et religieuses, n'a jamais figuré dans la liste des sujets de droit des gens ou de droit international. Seuls paraissent être compris dans une telle liste les Etats souverains et indépendants, les organisations internationales gouvernementales et dans certaines limites mêmes les individus, ressortissants, apatrides ou personnes physiques de multiples nationalités.

Admettre l'existence et même l'application du droit international dans un monde multiculturel ne semble pas poser de problèmes insurmontables. Mais une telle admission présuppose la reconnaissance des diverses cultures humaines ainsi que la coexistence juridique et pacifique parmi ces êtres humains de cultures différentes et même opposées ou au moins divergentes. La coexistence même d'une divergence ou diversité de cultures humaines ne signifie pas en elle-même et d'une façon automatique l'égalité que le droit est prêt à attribuer à chaque groupe de civilisation spécialisée, différente et non uniforme. Le passé ne porte aucun témoin de l'adoption dans la pratique des Etats d'un tel principe d'égalité, non pas parmi des Etats souverains et indépendants, mais plutôt parmi des êtres humains d'origines diverses et de cultures tout à fait divergentes.

Il serait utile d'admettre dès le début que le droit international tel que nous le concevons aujourd'hui a été le produit de la pratique des Etats européens dans un monde uniculturel, ou presque de culture unique et exclusivement chrétienne. Le bassin méditerranéen présentait un lien géographique entre l'Europe et l'Afrique et le 
Moyen-Orient ou l'Asie occidentale, à partir d'où fut connue la région d'outre-mer de l'Extrême-Orient comprenant le sous-continent, l'Asie du Sud-Est et l'Asie orientale. La découverte du Nouveau Monde a contribué à une libéralisation d'horizon terrestre et a servi à ouvrir la porte de connaissance de l'Amérique du Nord et de l'Amérique latine. Or, les deux continents américains ne semblaient guère diversifier les cultures qui s'étendaient de côte à côte à travers des deux continents, et de long en large, tout en dépassant et englobant, balayant et inondant ainsi les cultures traditionnelles indigènes préexistantes. Seuls les immigrés gouvernaient, étant tous représentants des cultures qui étaient presque toutes européennes et, par conséquent, toutes chrétiennes, qu'ils avaient introduites et importées avec eux au Nouveau Monde.

Certes, l'existence du droit international dans le monde ancien a été soutenue par plusieurs auteurs contemporains ${ }^{1}$. Le professeur Ago, par exemple, a conclu

«que le droit international dont nous avons hérité n'est que le produit concret des exigences réelles de la vie de relation qui a réuni, et ceci dès le début, une pluralité d'entités souveraines appartenant souvent à des civilisations différentes et, d'après le vocabulaire actuel, à des mondes distincts qui ont été amenés à se rencontrer au fil de l'histoire ${ }^{2}$.

Le droit international moderne, contemporain, n'est-il donc pas identifié avec le droit international de l'Ancien Monde? On risquerait d'avoir tort de ne pas prendre comme point de départ l'origine de la communauté internationale qui fut sans doute la communauté étatique européenne et chrétienne à la suite du Traité de Westphalie, et qui s'est progressivement étendue au cours des siècles pour atteindre le caractère universel actuel ${ }^{3}$. Hugo Grotius, qu'on a souvent appelé le père du droit international, à l'époque de la formation du droit des gens, suivant la transformation de la collectivité étatique internationale, cherchait à défendre la religion chrétienne dans son œuvre De veritate christianae religionis publiée en 1615. Même son chef-d'œuvre, De jure belli ac pacis, publié dix ans après, n'avait pas exercé trop d'influence hors des limites de la chrétienté. Dans son traitement du Mare liberum, ses mots introductifs furent adressés uniquement aux chrétiens: Ad principes populos que liberos orbis christiani ${ }^{4}$, aux princes et aux peuples libres du monde chrétien.
Même à cette époque la plupart des auteurs ont ignoré la coexistence en pratinne des anciens royaumes asiatiques comme le Siam (ou la Thailande, Pays des hommes libres), la Chine (ou l'Empire céleste) et le Japon (ou l'Empire du Soleil-Levant). Parmi ces collectivités étatiques, il y avait eu entré elles des contacts interétatiques et des rapports commerciaux et diplomatiques importants. Dans le temps même de Grotius, le Siam envoya sa première mission diplomatique aux Pays-Bas au début du XVII siècle et procéda à conclure le premier traité de commerce entre ces deux entités étatiques séparées par une distance gigantesque. Des missions di plomatiques furent échangées non seulement entre ces deux pays, dont l'un de l'Asie du Sud-Est et l'autre de l'Europe occidentale, mais aussi peu de temps après entre le roi Narai le Grand, du Siam, et le roi Louis XIV, de France, d'où la scène cérémoniale de présentation des lettres de créance au château de Versailles qui fit l'objet d'une peinture historique impressionnante pour des étudiants de l'histoire diplomatique et des relations internationales entre deux sociétés culturelles fondamentalement différentes. Ces rapports diplomatiques et historiques intercontinentaux, ou mieux encore européo-asiatiques, se sont déroulés à travers des siècles jusqu'à nos jours sans se détériorer ou se transformer en rapports de conquête ou d'expansion coloniale. Ces mêmes rapports, soutenons-nous, devraient se baser sur un fondement de droit, sur les mêmes principes universels, de droit des gens, malgré les différences, si profondes soient-elles, entre les deux cultures diverses, dont l'une chrétienne et l'autre bouddhique. C'est le seul cas de coexistence juridique et pacifique entre un Etat européen et un Etat asiatique sur pied de parfaite égalité à travers l'histoire de la civilisation humaine.

Pourtant, le droit international prendra encore beaucoup de temps et plusieurs siècles pour devenir véritablement de plus en plus universel, substantiellement de plus en plus humanitaire ou humanisé, et par la suite réellement de plus en plus tolérable sinon complètement acceptable.

On se demande si l'acceptation positive indiquant le consentement de l'Etat peut constituer une base juridique ou l'opinio juris d'une règle coutumière, ou s'il n'est pas absolument nécessaire qu'une règle de coutume internationale soit adoptée et acceptée dans la pratique générale des Etats avant qu'elle ne prenne ainsi force de droit ou avant qu'elle ne soit dotée d'un effet juridique obligatoire. La notion de tolérance que nous a enseignée le Bouddha 
suffit-elle pour impliquer sinon présumer ou manifester l'existence de l'assentiment ou pour en déduire les conséquences juridiques du zonsentement volontaire? Toutes ces questions sont pertinentes lans notre étude du développement progressif du droit internatioral. On est convaincu, avec la notion actuelle de l'égalité et de la ouveraineté de chaque Etat, que la tolérance n'équivaut pas accepation. Tolérer ne veut pas dire accepter, ni acquiescer. En réalité, a tolérance pacifiste ne peut être interprétée que comme une orme de politesse d'une expression de désapprobation tacite, et in nulle manière ne pourrait être considérée comme acquiescement su acceptation d'une telle ou telle règle ou d'une telle ou telle ratique ou usage coutumier.

Le consentement de l'Etat se fonde sur une base plus solide, car e droit devrait relever de l'expression de la conscience publique. ee droit, dont nous parlons, a déclaré Chao Phya Aphay Raja ${ }^{5}$,

«n'est pas un droit formel, littéral, procédant a priori, découpé en théorèmes ou en paragraphes dans les livres classiques, mais le droit nécessaire, historique, essentiellement progressif, dont la légitimité se fonde sur la nature des choses. C'est dans l'opinion ou la conscience publique qu'il faut chercher la première manifestation puissante de ce droit...»

Depuis très longtemps déjà, il nous a apparu que «le droit interational ne pourrait se dire véritablement humain, cosmopolite, ¡ue lorsque ses règles embrasseraient ce vaste monde », y compris ans doute le «monde oriental, auquel aujourd'hui nous touchons ar tant de côtés, sans le pénétrer par un seul » ${ }^{6}$. Déjà en 1876 , Thao Phya Aphay Raja fut d'avis que le droit international se doit 'englober l'Orient, mais celui-ci doit s'ouvrir à la civilisation occientale:

«Il y a pourtant, pour qu'il soit résolu pacifiquement, une condition essentielle à remplir: c'est qu'il y ait possibilité démontrée pour les Orientaux et les Occidentaux, pour les chrétiens, les mahométans, les hindous, les Chinois, les Japonais, etc., de s'établir réciproquement les uns chez les autres en jouissant pour leurs personnes et leurs propriétés d'une protection égale à celle qu'ils obtiennent dans leur pays

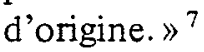

Dans l'appréciation de la valeur de la conscience juridique progressive de l'humanité, Chao Phya Aphay a observé en 1886 que,

«au milieu de ces migrations, de ces choses et de ces superpositions de races, la notion d'une unité humaine supérieure à la variété des groupes, notion obscure d'abord, puis de plus en plus distincte, se fait jour. Aujourd'hui déjà, les progrès de la conscience publique nous permettent d'affirmer comme une vérité incontestable que le développement et, par conséquent, la liberté rationnelle de l'être humain forment le but principal et légitime de tout droit, national ou international. Il en résulte que les différences de race ou de nationalité ne doivent être prises en considération par le droit international que si leur reconnaissance devait compromettre le libre développement de tout un groupe humain. ${ }^{8}$

Presque un siècle après, du temps de Chao Phya Aphay jusqu'à nos jours, cette notion d'universalité du droit international ainsi que son côté humain se voient fleurir, réitérés et réunis dans un concept plus récent, celui du patrimoine commun de l'humanité, étant

«le résultat d'une évolution qui ne laisse pas indifférent le droit international dans son ensemble; en fait, il y introduit des transformations révolutionnaires, qu'il s'agisse, notamment, de la reconnaissance de la qualité de sujet de droit à l'humanité en tant que telle ou de l'exploitation directe de ressources communes par un organisme international $\gg$.

La question se pose si l'humanité peut être sujet de droit international en tant que telle. On ne voit pas pourquoi l'humanité en tant que telle ne pourrait pas être sujet de droit international, titulaire de droits et tenue à des obligations selon le droit international ${ }^{10}$.

L'humanité n'est pas identifiée avec la communauté internationale qui est devenue institutionnalisée, tandis que l'humanité en tant que telle est composée de ses éléments humains, et ainsi constituée par la totalité ou l'entier des espèces humaines de toutes les races et civilisations. Ceci ne veut pas dire ou contredire que l'humanité ne pourrait être représentée par la communauté internationale. C'est bien la communauté mondiale qui devrait représenter l'humanité dans toute son étendue, dans toute son ampleur et dans 
outes ses manifestations. Par conséquent, les deux notions, ces eux expressions ne sont ni identiques ni remplaçables, car l'humaité en tant que telle est un élément constitutif de la communauté nternationale et mondiale ou de la collectivité interétatique, et ette dernière n'est investie que du caractère représentatif de l'hunanité, et ne pouvant agir qu'en tant que représentant de l'homme armi ses autres qualités représentatives.

L'humanité en tant que notion juridique distincte, une fois adnise comme sujet de droit international, se met à jouer plusieurs oles de plus en plus importants dans le développement progressif iu droit international.

En tant que sujet de droit, cette notion de l'humanité doit être ntendue et interprétée comme embrassant toutes les espèces de omo sapiens, n'importe laquelle et sans avoir égard aux races, regions ou distinctions sociales quelconques. La notion juridique e l'homme ou de l'humanité dans son entier devrait comprendre \& notion biologique et physique de l'homme. Autant qu'il y a tomme ou homo sapiens, on se trouve en présence d'un élément onstituant de l'humanité sans distinction de race, religion ou autre $\mathrm{t}$ sans discrimination aucune. La notion de l'homme doit s'entendre le la totalité de l'humanité et de tous les hommes, femmes et enants, composant l'humanité tout entière. L'expression autre que 'homme ne peut pas signifier l'humanité, ni l'expression citoyen, tomme libre, peuple, ressortissant, ni la notion de nationalité ou 'absence de celle-ci puisse altérer la portée juridique de la notion le l'humanité.

De par la nature de l'humanité en tant qu'élément signifiant et ontribuant au développement progressif du droit international ontemporain, on doit s'interroger sur les divers domaines de ses ontributions.

\section{Les pirates ex jure gentium: "hostes generis humani»}

Au premier abord, déjà à l'époque classique de la formation du Iroit international traditionnel ou même moderne, la notion de 'humanité n'était pas étrange au droit international. Elle se vit eflétée, adoptée et confirmée dans l'usage du vocabulaire du droit nternational contemporain. Ainsi, dans le domaine de piraterie $e x$ "re gentium ${ }^{11}$, comme crime ou délit grave de droit international ublic, les pirates ont été censés être ennemis de l'humanité. Les pirates étaient appelés "hostes generis humani», ennemis du genre humain, ennemis des gens, comme d'ailleurs l'expression le droit «des gens», signifiant «de la communauté internationale» ou interétatique dans son ensemble. C'est l'humanité en tant que telle qui est mise en cause par les activités des pirates en haute mer, et, par conséquent, quiconque est autorisé à arrêter les pirates pour les traduire en justice et afin de prendre toutes les mesures appropriées de punition, de répression ou de prévention. Ces criminels de droit international dans la conception classique de droit des gens que sont les pirates ex jure gentium, que tout Etat et toute autorité nationale ou internationale pourraient poursuivre en haute mer et en justice, devraient être traités cependant comme des êtres humains avec toutes les garanties de la justice et du droit, bien qu'ils soient ennemis du genre humain et dans ce sens de ses propres espèces humaines.

\section{Un code des crimes contre la paix et la sécurité de l'humanité}

En second lieu, l'humanité figure éminemment s'agissant du traitement des crimes contre la paix et la sécurité de l'humanité. La Charte des Nations Unies parle de «la paix et la sécurité internationales » qui ne sont pas identiques à la paix et à la sécurité de l'humanité, car la notion de l'humanité est beaucoup plus large et plus étendue que celle de la nation ou de la nationalité. Les hommes font partie de l'humanité quoiqu'ils ne fassent pas partie d'une nation quelconque et bien que dépourvus de toute nationalité et de tout lien avec un Etat quelconque. Il s'agit de la responsabilité des individus ainsi que de celle des Etats pour les crimes commis par eux, individus comme Etats, contre la paix et la sécurité de l'humanité, ce qui est différent de la question du maintien de la paix et de la sécurité internationales. Il peut y avoir le maintien de la paix et de la sécurité internationales sans que la paix et la sécurité de l'humanité soient préservées. Or, on peut porter atteinte à la paix et à la sécurité de l'humanité sans porter aucun préjudice à la paix et à la sécurité internationales. La dignité de l'homme peut faire l'objet d'une atteinte sévère sans troubler dans la moindre mesure la paix et la sécurité internationales. Un Etat ou une société humaine peut décider de traiter ses membres d'une manière inhumaine. Il peut y avoir une persécution contre un groupe de minorités religieuses, raciales, politiques ou sociales à l'intérieur d'un Etat 
;ouverain sans déborder ses frontières nationales, sans gêner dans uucun sens la paix et la sécurité internationales. Tout cela n'emjêche pas qu'un crime soit commis contre la paix et la sécurité de 'homme, tel que le génocide ou l'apartheid.

Dans l'attente d'une codification complète des crimes contre la paix et la sécurité de l'humanité ${ }^{12}$, il faut compter parmi ces crimes de droit international:

1) tout acte d'agression contre un autre Etat;

2) toute menace de recourir à un acte d'agression contre un autre Etat;

3) le fait de préparer l'emploi de la force armée contre un Etat;

4) le fait d'organiser ou d'encourager l'organisation de bandes armées en vue d'incursions sur le territoire d'un autre Etat, ou d'en tolérer l'organisation sur son propre territoire;

5) le fait d'entreprendre ou d'encourager les activités visant à fomenter la guerre civile dans un autre Etat;

6) le fait d'entreprendre ou d'encourager des activités terroristes dans un autre Etat, ou de tolérer des activités organisées calculées en vue de perpétrer des actes terroristes dans un autre Etat;

7) les actes commis en violation des obligations qui incombent à l'Etat en vertu d'un traité destiné à assurer la paix et la sécurité internationales au moyen de restrictions et de limitations aux armements, à la préparation militaire ou aux fortifications;

8) le fait d'annexer, au moyen d'actes contraires au droit international, un territoire appartenant à un autre Etat;

9) le fait d'intervenir dans les affaires intérieures ou extérieures d'un autre Etat par des mesures de coercition, d'ordre économique ou politique;

10) les actes commis dans l'intention de détruire un groupe national;

(1) les actes inhumains, tels que l'assassinat, l'extermination, la réduction en esclavage, la déportation ou les persécutions commis contre des éléments de la population civile pour des motifs sociaux, politiques, raciaux, religieux ou culturels;

(2) les actes commis en violation des lois et des coutumes de la guerre;

3) les actes constituant le complot, l'incitation directe, la complicité dans l'un quelconque des crimes ainsi définis, et la tentative pour commettre l'un de ces crimes.
A cette liste s'ajoutent les crimes connus comme l'apartheid, le génocide et les crimes contre l'environnement.

Dans l'analyse finale, ces crimes ont pour but commun l'atteinte à l'humanité, à la paix de toute l'humanité et à la sécurité de l'humanité tout entière.

\section{Le caractère humanitaire du nouveau droit international}

Si l'humanité ne faisait pas partie du droit international classique et traditionnel, si l'homme n'avait pas de statut juridique aux yeux du droit des gens de jadis, il en est autrement aujourd'hui. Plus le droit international moderne s'applique à tout le monde, à tous les pays, plus on reconnaît l'universalité de l'application du système unique de droit international et plus on continue à introduire et à renforcer le caractère humanitaire de ce droit. Le droit, comme toute autre chose, devrait être humanisé, aussi longtemps que l'on garde l'espoir de survivre, face au danger d'une troisième guerre mondiale, qui sera la dernière, et aux risques de pollution de l'environnement dans lequel nous vivons sur terre, en haute mer et dans l'atmosphère circumterrestre.

Si les Etats nouvellement indépendants, qui sont tous ou presque tous des pays en voie de développement, ont hésité à accepter immédiatement et sans aucune réflexion les règles existantes du droit coutumier ou des coutumes internationales, c'était parce que le droit international tel qu'il a évolué pendant les quelques siècles précédents a montré un manque visible d'élément humain, de caractère humaniste et de principes humanitaires. Les aversions que ces nouveaux Etats ont éprouvées contre le droit classique et traditionnel sont fondées non seulement sur des soupçons intuitifs ou instinctifs, mais aussi sur une base solide, d'inégalité, d'injustice et de faute inhérente à ce droit de respect dû à l'humanité.

Petit à petit, la confiance des nouveaux Etats commence à s'accroître, suivant l'adoption de la résolution 1514 sur l'octroi de l'indépendance, la reconnaissance du jus cogens qui servira à annuler les accords inhumains, les accords d'asservissement ou d'esclavage, et l'impossibilité de rétablir un régime colonial quelconque ou de conquête ou de subjugation d'un peuple par un autre. Ces nations dites civilisées qui sont responsables de ces injustes régimes ont commencé à comprendre les aspirations de l'homme, de sa dignité 
t de sa propre place, que tous les Etats devraient accorder à l'hunanité au sein même du droit international.

Le caractère humanitaire du droit international s'est révélé sous eux aspects différents mais profondément et étroitement liés, à avoir, les droits de l'homme et le droit international humanitaire. fistoriquement et paradoxalement, le droit international humaniaire a évolué et s'est développé à travers le droit de la guerre ou es règlements humanitaires pour réglementer les actes de guerre, ¿s batailles, les traitements des prisonniers de guerre, des blessés et es malades. C'était sous cet angle que l'élément humanitaire de roit international fut reconnu et renforcé, non encore pour élimier et éviter la guerre, mais simplement pour en réduire les conséuences nuisibles et les souffrances soutenues par les militaires ainsi ue par les civils. Les Règlements de La Haye avaient pour but la églementation de faire la guerre, d'éliminer les souffrances inutiles t évitables. Les Règlements de La Haye de 1899 et de $1907^{13}$ ont té renforcés par les Conventions de Genève de $1949^{14}$ et les Proocoles additionnels aux Conventions de Genève de $1977^{15}$.

Le droit international ainsi humanisé après l'extension de son pplication universelle devrait être encore plus modernisé. Si homme devait son existence continue à l'homme, il devrait sa isparition totale à personne d'autre qu'à lui-même. Si l'homme st actuellement investi du droit d'autodétermination garanti par 1 Charte et la Déclaration universelle des droits de l'homme, il est galement capable de l'auto-extermination. Dans ces conditions, paraît absolument indispensable que l'homme soit empêché de 3 démolir en tant qu'espèce humaine. La guerre n'est plus consiérée comme moyen licite de politique nationale. Il n'existe plus e règle de droit permettant le droit de prendre la vie d'un être umain. Même l'ennemi tombé n'est pas obligé à se soumettre à exercice du jus vitae necisve. Il n'existe plus de droit de conquête u de gaspillage des biens, intérêts et avoirs des peuples subjugués $u$ assujettis à l'esclavage permanent.

L'autre côté humanitaire du droit international s'est manifesté ans le domaine des droits de l'homme, des libertés fondamentales e l'homme. Ces droits sont traduits en pratique sous forme d'obliations des Etats de reconnaître et de donner suite aux droits et bertés qui appartiennent à tous les êtres humains en tant que tels non pas en qualité de citoyens, ou ressortissants ou peuples ivilégiés.
Chaque être humain est né libre et ne peut nulle part être mis aux chaînes sauf pour les actes dont il est personnellement responsable. La Déclaration universelle des droits de l'homme de $1948^{16}$ a été suivie par le Pacte international relatif aux droits économiques, sociaux et culturels (1966) ${ }^{17}$, le Pacte international relatif aux droits civils et politiques $(1966)^{18}$ et le protocole facultatif se rapportant à ce pacte $(1966)^{19}$. Ces documents constituent la Charte internationale des droits de l'homme. En outre il y a bien d'autres instruments internationaux touchant aux divers aspects des droits de l'homme, y compris le droit à l'autodétermination, la non-discrimination raciale, la prévention des crimes contre l'humanité, y compris le génocide, la Convention relative à l'esclavage, etc. ${ }^{20}$

\section{Le patrimoine commun de l'humanité}

Le droit international tel qu'il a été ainsi humanisé, tout en incorporant l'élément humanitaire, y compris les droits de l'homme et le droit humanitaire, fait partie du patrimoine incorporel de l'humanité. Le patrimoine corporel de l'homme dans son ensemble est composé de plusieurs parties: du patrimoine céleste, comprenant les espaces extra-atmosphériques, la Lune et les corps célestes, et terrestre, comprenant l'espace aérien, l'environnement circumterrestre et le domaine terrestre au-delà du territoire ou de la juridiction nationale d'un Etat. En outre il faut citer aussi l'exemple du patrimoine culturel de l'humanité, y compris, tous les biens culturels, tels que les monuments, les archives, les objets d'art de valeur historique, les temples, les églises, les tombeaux, ainsi que les biens naturels, et aussi les propriétés intellectuelles ou industrielles, comme les droits d'auteur, les brevets d'invention, les droits littéraires et musicaux. La science et la technologie font partie du patrimoine culturel et scientifique de l'homme.

La notion est encore évolutive mais l'humanité figure très clairement en occupant la place d'honneur en tant que titulaire du patrimoine commun ou mondial de l'homme ${ }^{21}$. Sauf en ce qui concerne le régime des fonds marins, réglé par le nouveau droit de la mer, et le régime spécial de la Lune et les autres corps célestes ainsi que les espaces extra-atmosphériques, le droit international sur le sujet de patrimoine commun de l'humanité est en train de se cristalliser et de se transformer en passant du domaine de lege ferenda au domaine de droit conventionnel ou de lex lata. 


\section{Conclusions générales}

En concluant cette brève étude sur l'humanité dans le contexte e l'avenir du droit international dans un monde multiculturel, uelques observations générales semblent justifiées.

$\mathrm{Au}$ fur et à mesure que le droit international étend son applicaon dans le monde toujours multiculturel, de plus en plus les voies ouvrent pour la participation de tous les groupes humains à la daction et à l'adoption des règles de droit international. Plus on xamine la portée élargie de l'application du système juridique nique au monde entier, plus on est convaincu que la conscience ablique l'emportera finalement au service et au profit de l'humaté tout entière. Ni le droit ni son développement progressif ne ourra plus rester propriété exclusive de certains groupes de peuples uropéens, fidèles à quelques religions chrétiennes. Le droit des gens jit être véritablement internationalisé et dans ce sens humanisé on seulement dans son application mais, plus important encore, ans son évolution toujours améliorante. Sinon on risquerait de 'éer ou tolérer l'application continue des règles de droit t $\epsilon$ :dant à śnéficier uniquement à certains secteurs de l'humanité au détrient du reste des êtres humains qui, à plus forte raison, auraient ssoin du concours du droit international, et qui mériteraient ailleurs sa protection.

Le passé a été si cruel pour la plupart de l'humanité. L'avenir ne surra pas répéter ou tolérer les erreurs du temps passé. Le problème sentiel est celui de l'enseignement fondamental et de l'éducation imordiale pour faire entendre que l'homme soit défini comme mmo sapiens par sa propre physique, basée sur des données pureent biologiques, sans exclure quelques espèces du genre humain uur des raisons ou des motifs de races, religions, politiques ou tuations économiques ou financières. Ces différences ou même fériorité ou supériorité de races, etc., ne peuvent ni altérer ni odifier ni élever le statut ou la dignité de l'humanité, d'un être imain, avec tous ses droits de l'homme et toutes ses libertés fonimentales ainsi que toutes ses fautes humaines.

Dans ces conditions et dans des domaines qui ont fait l'objet de tre enquête, l'homme en tant que tel, comme l'humanité dans in entier, a contribué positivement et devrait continuer à appor$r$ ses contributions substantielles aux développements progresfs et incessants du droit international contemporain dans une collectivité mondiale multiculturelle et pourtant humanisée sinon civilisée.

En dernier lieu, ce serait l'homme lui-même, avec tous ses défauts et imperfections, avec toutes ses qualités humaines et inhumaines, qui serait placé en mesure de se détruire et d'introduire dans ce monde un tel holocauste global et total. Ce serait également grâce à l'homme que l'humanité tout entière pourrait et devrait être sauvee de ce désastre imminent. Car sans l'humanité il ne peut exister ni Etat, ni communauté internationale, ni droit international pour régler entre ces entités nationales les différends interétatiques et les rapports internationaux.

Avec l'aide de l'humanité et de l'élément humain du droit international, le droit devrait progresser et évoluer sans perdre de vue sa principale composante, à savoir la société humaine. L'homme en tant que tel doit être reconnu en droit international et l'humanité tout entière devrait se faire valoir comme sujet de droit dans ce monde multiracial et par conséquent multiculturel. Le principe de la non-discrimination se fera jour dans la pratique universelle des Etats. Le jour viendra dans un avenir non lointain où l'humanité triomphera si de tout le genre humain viendra le sauvetage.

Il faut rappeler néanmoins que chez les bouddhistes rien n'est permanent. Tout passe avec le cours du temps. Toute chose mondaine et terrestre est temporelle. Tout être humain est mortel. L'homme naît, vieillit, tombe malade et meurt à un moment donné. Le droit international en tant que création humaine, comme l'humanité elle-même, est conçu, mis en application, adopté, vénéré, et, tout d'un coup, abandonné, il tombe en désuétude et disparaît sans trace, comme le corps humain, las, fatigué, âgé, malade, et un jour prêt à partir, mourir et éventuellement pourrir sans la moindre possibilité de résurrection.

Nous sommes persuadés que l'humanité en toute sagesse saura reporter sa fin à une date ultérieure au lieu de l'accélérer par sa propre impatience. Si par ailleurs l'homme persiste dans sa prudence, l'humanité aura encore sa chance. Son avenir peut être assuré tout en contribuant au développement bénéfique et utile du droit international pour renforcer le statut juridique de l'homme comme sujet de droit et pour ainsi augmenter son rôle responsable et respectable dans le monde entier. 


\section{NOTES}

1. Voir par exemple, Paradisi, Storia del diritto internazionale nel Medio voo I, Milan, 1940 , pp. 8 ss., 261 , et «I fondamenti storici della comunità giuridica internazionale", dans Civitas Maxima. Studi di storia di diritto intergiurionale, I, Florence, 1974 , pp. 33 ss.

2. Roberto Ago, Mélanges offerts à Paul Reuter. Le droit international: nité et diversité, "Les premières coilectivités interétatiques méditerranéennes ", pp. 3-4; Verosta, «Die Geschichte des Völkerrechts », dans Verdross, Ölkerrechts, 5 éd., Vienne, 1964 , p. 32.

3. Ago, "Pluralism and the Origins of the International Community », ItaIian Yearbook of International Law, vol. III, Naples, 1978, pp. 3 ss.

4. Voir «Hugo Grotius, The Father of International Law», Conference on Deep Seabed, Mining and Freedom of the Seas, par Federich Tse-Shyang Chen, 18

5. Voir «Le droit international et la phase actuelle de la question d'Orient , our la vie de Chao Phya Aphay Raja (Gustave Rolin-Jaequemyns) voir, The Journal of Siam Society, vol. LIII, partie 2, 2 juillet 1965; Revue de droit international et de législation comparée, 1876, pp. 294 et 380. Chao Phya Aphay fut le principal conseiller du roi Rama $\mathrm{V}$ de Thaillande.

6. "Le droit international et la phase actuelle de la question d'Orient», Revue de droit international et de législation comparée, 1876, p. 293.

7. Lettre de Chao Phya Aphay Raja au docteur Dutrieux, au Caire, sur la question judiciaire en Egypte, ibid., 1876, p. 577.

8. "Les principes philosophiques du droit international - examen critique du système de M. Lorimer», Revue de droit international et de législation comparé, 1886 , p. 58

9. S. Bastid, "L'état du droit international public en 1973 », Journal du droit international, 1973 , pp. 5-21.

10. A.-C. Kiss, "La notion de patrimoine commun de l'humanitê"», Recueil des cours de l'Académie de droit international, 1982, tome 175, pp. 103-256.

11. Voir, par exemple, l'affaire In re Piracy Jure Gentium [1934] A.C. 586 , la page 600 ; Grotius, De jure belli ac pacis, vol. II, cap. 20,40

12. Voir dans ce sens le rapport de la Commission du droit international sur les travaux de sa trente-cinquième session, $A / 38 / 10$, chap. II, pp. 14-15; A/CN.4/85, Annuaire de la Commission du droit international, 1954, vol. II, par. $48-53$, pp. $150-151$, art. 2.

13. Le droit de La Haye, ou droit de la guerre, sert à définir et préciser les droits et devoirs des belligérants dans la conduite des opérations et limite le choix des moyens de nuire. Ces stipulations résultent, au principal, des Conventions de La Haye de 1899, révisées en 1907. Le droit de La Haye embrasse aussi des conventions comme celle de Saint-Pétersbourg de 1868 , interdisant les balles explosives, et le Protocole de Genève de 1925, prohibant l'emploi des gaz asphyxiants, des moyens bactériologiques et similaires.

14. Le droit de Genève, ou le droit humanitaire proprement dit, tend à sauvegarder les militaires mis hors de combat, ainsi que les personnes qui ne participent pas aux hostilités. Voir les quatre Conventions de Genève du 12 août 1949 .

15. Les Protocoles additionnels no $I$, relatif à la protection des victimes des conflits armés internationaux, et no II, relatif à la protection des victimes de conflits armés non internationaux, du 10 juin 1977 .

16. Adoptée et proclamée par l'Assemblée générale dans sa résolution $217 \mathrm{~A}$ (III) du 10 décembre 1948
17. Adopté et ouvert à la signature, à la ratification et à l'adhésion par l'Assemblée générale dans sa résolution $2200 \mathrm{~A}$ (XXI) du 16 décembre 1966 et entré en vigueur le 3 janvier 1976, conformément aux dispositions de l'article 27.

18. Adopté le même jour dans la même résolution $2200 \mathrm{~A}$ (XXI) du 6 décembre 1966; et entré en vigueur le 23 mars 1976, conformément aux dispositions de l'article 49 .

19. Ibid., entré en vigueur le 23 mars 1976, conformément aux dispositions de l'article 9.

20. Voir l'étude établie par Aureliu Cristescu, F/CN.4/Sub.2/404 Rev.1.

21. Voir mon ouvrage intitulé Une notion nouvelle: le patrimoine commun de l'humanité, sous préparation, Unesco; et A.-C. Kiss, "La notion de patrimoine commun de l'humanité", Recueil des cours de l'Académie de droit international, 1982 , tome 175 , pp. 103-256. 